\title{
Lateral subluxation of the atlas in rheumatoid arthritis: a case report and post-mortem study
}

\author{
N. BOGDUK, ${ }^{1}$ G. A. C. MAJOR ${ }^{2}$ AND J. CARTER ${ }^{2}$ \\ From the ${ }^{1}$ Department of Anatomy, University of Queensland, St Lucia, and the ${ }^{2}$ Department of Rheumatology, \\ Royal Newcastle Hospital, Newcastle, Australia
}

SUMMARY The atlantoaxial joints of a patient with lateral subluxation of the atlas were studied post mortem by dissection. There was severe erosion of the left lateral atlantoaxial joint and an asymmetrical erosion of the cartilages of the median atlantoaxial joint which prevented reduction of the lateral subluxation. The atlantoaxial ligaments were remarkably intact. It is emphasised that osseous factors rather than ligaments confer lateral stability to the atlantoaxial joints and that destruction of these leads to lateral subluxation.

Whereas there is an abundant and long-standing literature on ventral and rotatory subluxation. of the atlas, it is only recently that lateral subluxation of the atlas has been recognised as a complication of rheumatoid arthritis. In 1978 Burry et al. ${ }^{1}$ described three cases and Bunton et al. ${ }^{2}$ a further 2, while Corrigan ${ }^{3}$ had briefly mentioned the condition some years earlier.

Despite this clinical interest the exact pathomechanics of lateral subluxation have remained obscure. Bunton et al. ${ }^{2}$ argued that erosion of the lateral mass of the atlas with detachment of the transverse ligament was the principal underlying lesion, but there has been no direct demonstration of this.

In view of the absence of any previous pathological studies an opportunity was taken to examine the atlantoaxial joints of a patient, known to have lateral subluxation, after his death from respiratory complications of rheumatoid arthritis.

\section{Case history}

The patient was a 56-year-old male with a history of rheumatoid arthritis starting in 1960 . Initially only his left shoulder was affected, but subsequently the disease progressed to give a symmetrical polyarticular pattern of involvement associated with subcutaneous nodules and marked pulmonary fibrosis.

Accepted for publication 6 April 1983.

Correspondence to Dr N. Bogduk, Department of Anatomy, University of Queensland, St Lucia 4067, Australia.
Continuous active arthritis led to increasing deformities of the hands, feet, and knees. In 1971 a Fowler's procedure was performed to correct foot deformities, and the patient also underwent excision of nodules from the elbows and synovectomy of the right knee. Subsequently his complaints of joint pain and stiffness essentially settled, and the remaining problems were related mainly to the existing deformities.

Attention was first drawn to the neck in 1979 when an abnormal head position with recession of the left sternocleidomastoid muscle was noted. Neck movements were normal and there were no signs of spinal cord or nerve compression. Symptoms related to the neck consisted of only mild upper cervical pain on flexion of the neck, which the patient reported as having been present for some 4 years. Tomograms of the atlantoaxial joints revealed erosion of the left joint and subluxation of the atlas to the right (Fig. 1). No specific treatment was undertaken apart from advice to use a cervical pillow at night.

In January 1980 the patient developed a respiratory tract infection and died in respiratory failure. Post-mortem examination revealed bilateral suppurative pneumonia with chronic interstitial pneumonia, fibrosis and rhe umatoid changes in both lungs, congestive cardiac failure, chronic venous congestion of the liver, benign nephrosclerosis, and rheumatoid arthritis with active changes in subcutaneous nodules. The cervical vertebral column was removed en bloc for anatomical study. 


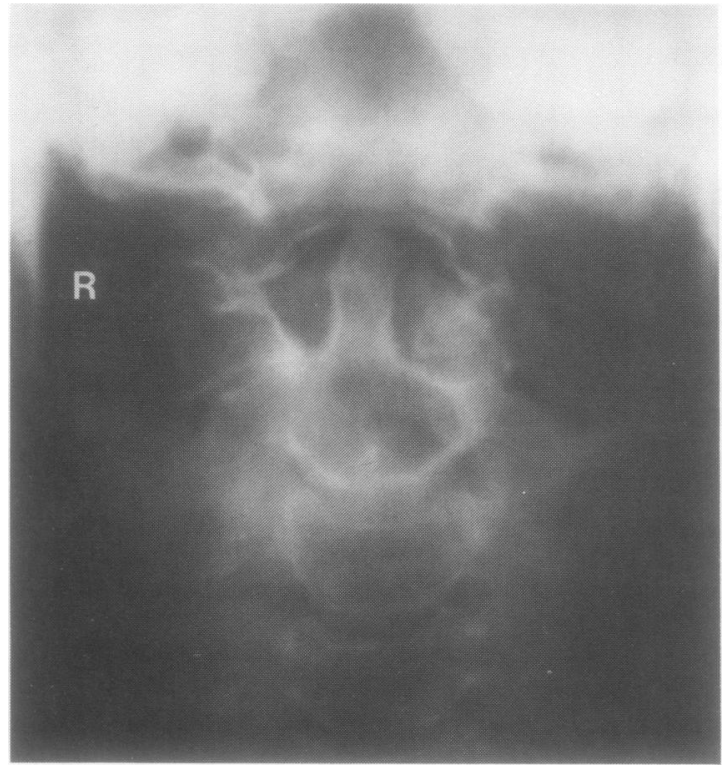

Fig. 1 Ante-mortem tomogram showing destruction of the le fi lateral atlantoaxial joint and subluxation to the right of the right atlantoaxial joint.

\section{METHODS}

The specimen obtained at post-mortem examination consisted of all 7 cervical vertebrae, though portions of the atlas were damaged during dislocation of the atlantooccipital joints. The specimen was fixed by immersion in $10 \%$ formalin. All the muscles attaching to the specimen were carefully resected without damaging any ligaments or joints.

Under a dissecting microscope when necessary the following structures were resected in turn: the posterior arch of the atlas, the anterior and lateral parts of the capsules of the lateral atlantoaxial joints, the membrana tectoria, the transverse ligament of the atlas, the anterior atlantoaxial membrane, the remainder of the capsules of the lateral atlantoaxial joints, and finally the capsule of the median atlantoaxial joint (atlantoodontoid joint).

At each stage of the dissection the extent of any subluxation was measured and the specimen tested manually for reducibility of this subluxation.

\section{Results}

On inspection of the intact specimen the atlas showed a $10^{\circ}$ tilt to the left and a $5^{\circ}$ rotation to the right. The anterior tubercle of the atlas was displaced $5 \mathrm{~mm}$ to the right of the midline (Fig. 2). Since the arthritic left lateral atlantoaxial joint offered no reliable landmarks, the extent of any subluxation was measured only in the right lateral atlantoaxial joint. A $5 \mathrm{~mm}$ lateral overlap of the margins of this joint was confirmed by dissection (Figs. 2, 3, 4).

The subluxation was a true lateral subluxation and not due to rotation of the atlas. It could not be reduced by rotatory or sagittal movements of the atlas, nor could it be reduced by pushing the atlas directly to the left. There was no ventral subluxation of the atlas.

The left lateral atlantoaxial joint was filled with haemorrhagic pannus which formed adhesions between the atlas and axis. No articular cartilage remained, and the bony facets were severely eroded. The erosion was pronounced anteriorly, where destruction of the superior articular process of the axis had allowed lowering of the left lateral mass, which accounted for the left lateral tilt and slight right rotation of the atlas (Fig. 2). Posteriorly erosion of the articular facets left only craggy bony surfaces (Fig. 3). In keeping with the subluxation to the right, the left lateral mass approximated to and was impacted against the base of the odontoid process (Fig. 4).

The right lateral atlantoaxial joint contained a healthy-looking synovial fat pad but no pannus. Its articular cartilages were reasonably intact, showing only a few small areas of partial-thickness erosion.

The capsules of the lateral atlantoaxial joints were grossly intact. Their resection permitted free rotation of the atlas but not reduction of the lateral subluxation.

The membrana tectoria and the transverse ligament of the atlas were grossly intact, the latter being

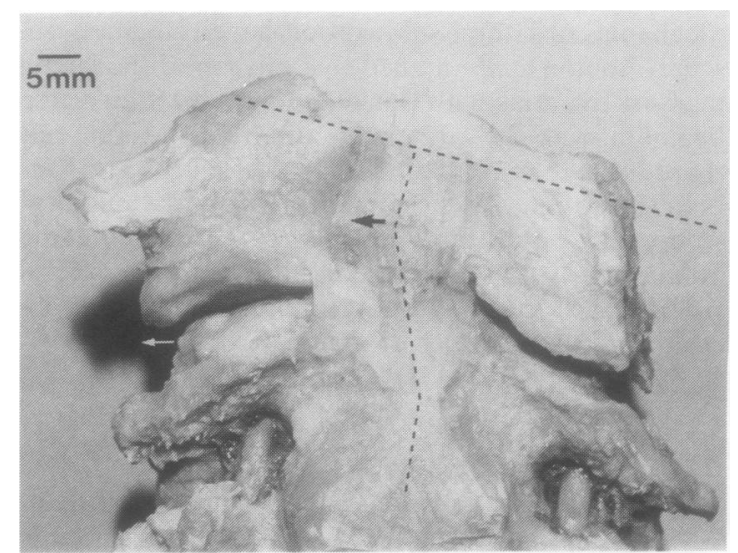

Fig. 2 Anterior view of the dissected specimen. The capsules of the lateral atlantoaxial joints have been resected to reveal the internal features of the joints. Note the lower position of the left lateral atlantoaxial joint; the $10^{\circ}$ tilt of the atlas to the left; the $5 \mathrm{~mm}$ lateral displacement of the anterior tubercle of the atlas; and the $5 \mathrm{~mm}$ lateral subluxation of the right lateral atlantoaxial joint. 


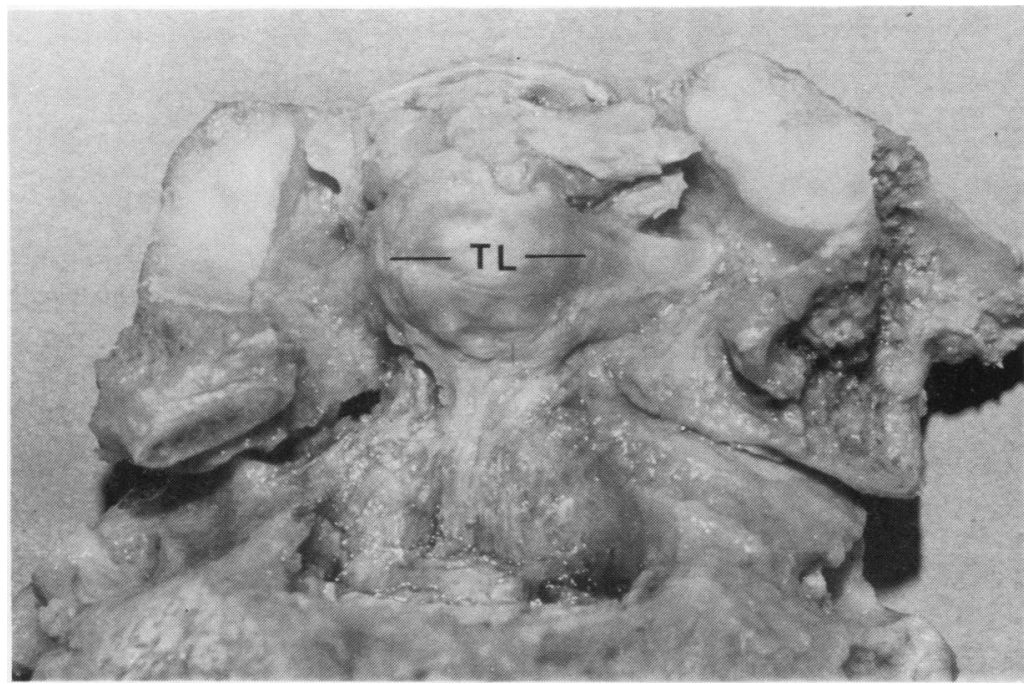

Fig. 3 Posterior view of the dissected specimen. The membrana tectoria has been removed to reveal the intact transverse ligament (TL). The apparent asymmetry of the ligament is due to the left band of the ligament having been approximated to the odontoid process by the lateral subluxation and slight rotation of the atlas.

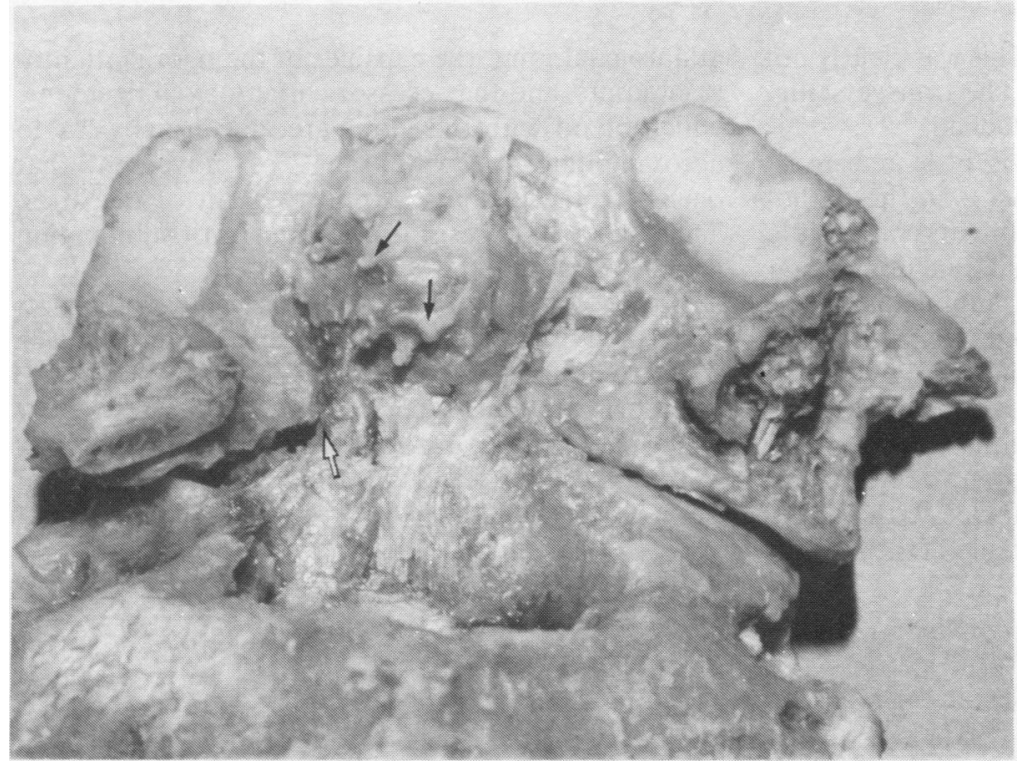

Fig. 4 Posterior view of the dissected specimen after removal of the transverse ligament. Note the integrity of the odontoid process, the array of spikes emanating from its posterior surface (small arrows); and the impaction of the left lateral mass of the atlas against the odontoid process (white arrow).

quite thick and still strongly attached to the lateral masses of the atlas (Fig. 3). Resection of these ligaments did not accentuate the lateral subluxation, but nor did it permit reduction of the subluxation.

Removal of the transverse ligament revealed a fascinating array of bony spikes emanating from the back of the odontoid process (Fig. 4). The transverse ligament bore on its anterior surface several reciprocal deep sockets which lodged these spikes.

Reduction of the lateral subluxation was not achieved until after the capsule of the median atlantoaxial joint was finally resected. This step revealed the factor which had prevented reduction.
The articular cartilage of the median atlantoaxial joint had been eroded asymmetrically, producing a joint space set obliquely forwards and to the left (Fig. 5 ). The atlas, having shifted to the right, could not be replaced because of impaction of the persisting articular cartilages. Resection of the median atlantoaxial joint capsule allowed the articular surfaces to be disengaged and the subluxation to be reduced.

\section{Discussion}

Previous interpretations of the pathomechanics of lateral subluxation implicated detachment of the 


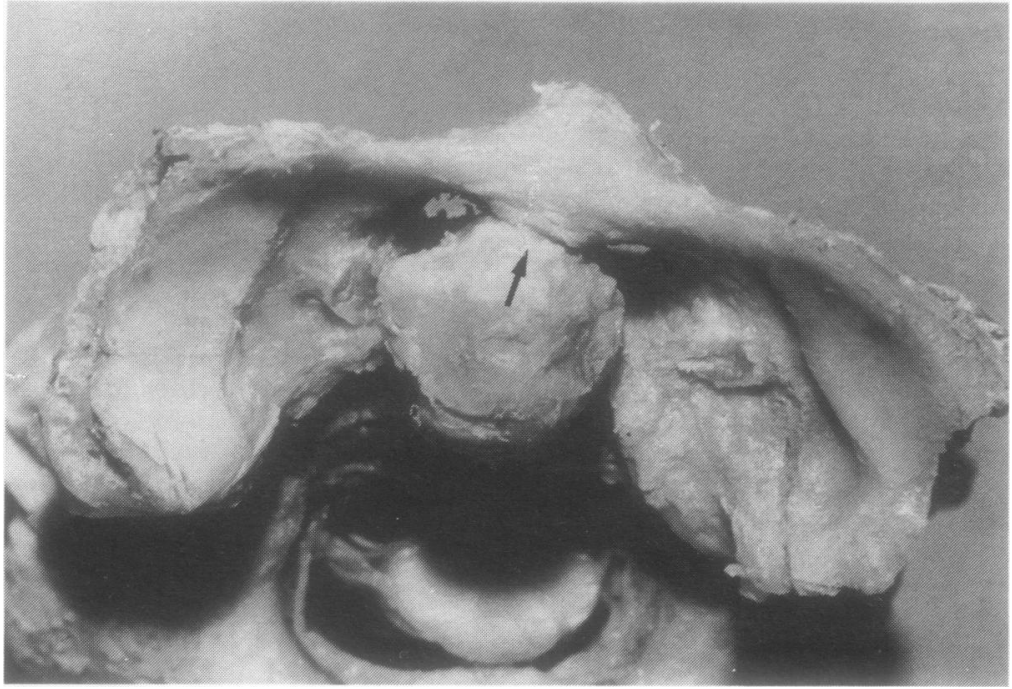

Fig. 5 A top view of the median atlantoaxial joint (arrow) showing the obliquity of the joint cavity caused by asymmetrical erosion of the articular cartilages, which prevented reduction of the lateral subluxation.

transverse ligament of the atlas. ${ }^{2}$ This was clearly not the case in the present specimen. The transverse ligament was robust and strongly attached.

On anatomical grounds there is little reason to implicate damage to major ligaments in the pathogenesis of lateral subluxation. Ligaments confer little lateral stability to the atlantoaxial junction. Their principal role is to resist ventral subluxation. The transverse ligament spans from one lateral mass of the atlas to the other, and is only indirectly attached to the axis through the capsule of the small transverso-odontoid joint. The longitudinal band of the cruciate ligament and the membrana tectoria are oriented rostrocaudally, at right-angles to the direction of lateral subluxation. The capsule of the median

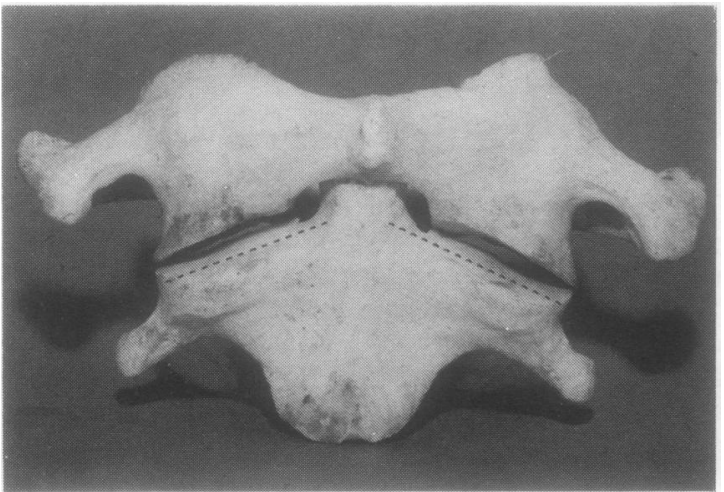

Fig. 6 Anterior view of a normal axis and atlas showing the obliquity of the lateral atlantoaxial joints, which is one of the osseous factors which impede direct lateral translation of the atlas. atlantoaxial joint, the capsules of the lateral atlantoaxial joints, and the accessory atlantoaxial ligaments which blend with the latter are all normally lax to accommodate the wide range of movement that occurs at these joints during rotation of the atlas. Thus they would resist only extremes of subluxation in any direction.

It is osseous features which principally confer lateral stability to the atlas, and destruction of these factors would predispose to lateral subluxation. First, in a normal atlantoaxial joint the superior articular processes of the axis are symmetrically sloped downwards and laterally and the atlas rests on this bilateral slope (Fig. 6). The slope impedes direct lateral

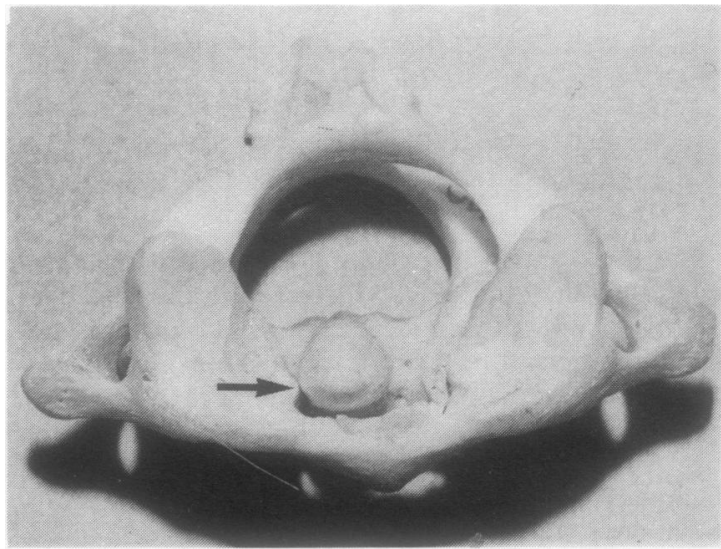

Fig. 7 Superior view of a normal axis and atlas illustrating how lateral subluxation would be limited by impaction of the contralateral lateral mass of the atlas against the base of the odontoid process. 
translation of the atlas, which can occur only if the atlas rides up the slope on one side and down the other. Secondly, there is a finite limit to the extent of any lateral subluxation. If the atlas moves sideways the contralateral lateral mass will eventually impact against the odontoid process (Fig. 7). The extent of any lateral subluxation is thereby restricted to the distance between the medial aspect of the lateral mass and the odontoid process, which normally is within the vicinity of $5 \mathrm{~mm}$.

Destruction of a lateral atlantoaxial joint is the critical lesion which allows lateral subluxation to occur clinically. Erosion of at least one lateral atlantoaxial joint was the lesion common to all the cases described by Burry et al. ${ }^{1}$ and Bunton et al. ${ }^{2}$ as well as the present case.

A

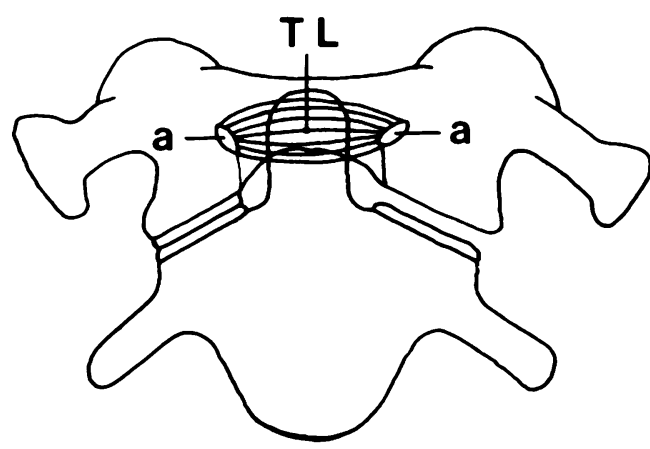

i.

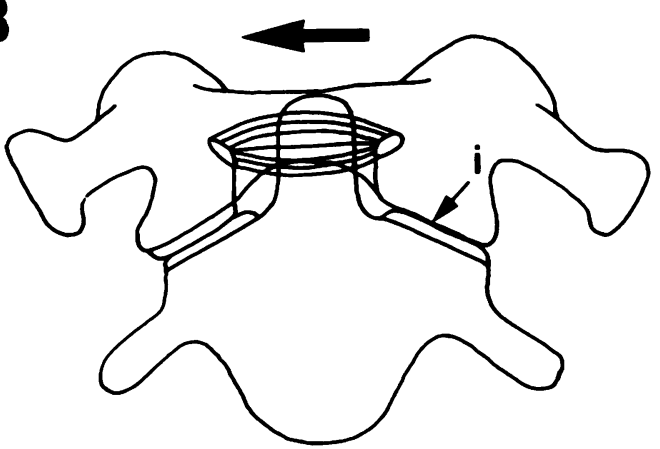

If cartilage and bone are eroded from either the superior articular process of the axis or the inferior articular process of the atlas, then the joint space is effectively widened and the articular processes no longer impede lateral movement of the atlas (Fig. $8 \mathrm{~A})$. If the erosion is slight, the lateral translation will eventually be arrested by the impaction of the remnants of the articular processes (Fig. 8B). In cases of greater erosion the articular processes, because of their reduced size, do not impact, and lateral translation is limited by the ultimate impaction of the lateral mass of the atlas against the odontoid process (Fig. 8C). In this respect it is noteworthy that impaction of the lateral mass against the odontoid process was observed in each of the cases described by Burry et al. ${ }^{1}$ as well as in the present case.

C
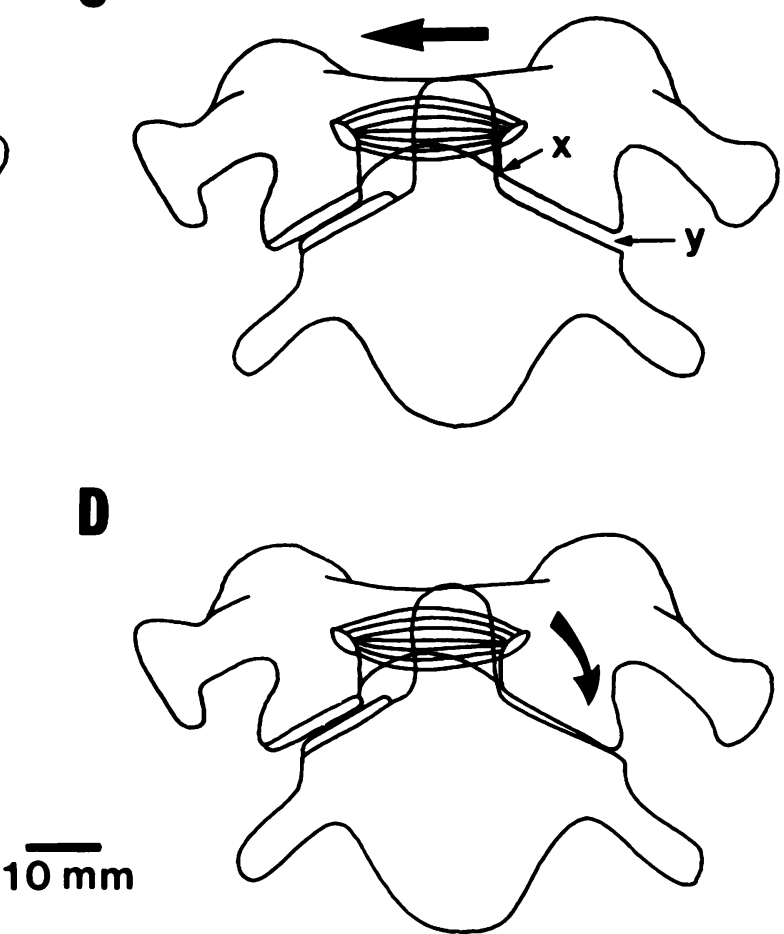

Fig. 8 The mechanism of lateral subluxation of the atlas. Each illustration is a silhouette of the margins of the axis and atlas viewed from the front. Also shown are the transverse ligament of the atlas (TL) and its attachment (a) to the medial aspect of each lateral mass. A: A specimen with full-thickness cartilage erosion and less than $1 \mathrm{~mm}$ bony erosion of the left inferior articular process of the atlas. The eroded lateral atlantoaxial joint offers no resistance to direct lateral translation. B: Lateral translation is arrested by the impaction (i) of the remnants of the articular processes. The right lateral atlantoaxial joint exhibits $2.5 \mathrm{~mm}$ of lateral subluxation. C: A specimen with full-thickness cartilage erosion and $1 \mathrm{~mm}$ of bony erosion of both articular processes of the left lateral atlantoaxial joint. Upon lateral translation the articular processes do not impact (y) and displacement is limited by impaction of the lateral mass against the odontoid process $(\mathbf{x})$. The right lateral atlantoaxial joint exhibits $4.5 \mathrm{~mm}$ of lateral subluxation. The eroded lateral atlantoaxial joint does not support the atlas (y) which therefore settles into the eroded joint undergoing a lateral tilt, D. The attachments of the transverse ligament lie some $4 \mathrm{~mm}$ above the lateral atlantoaxial joint space and are not involved in erosive changes sufficient to permit lateral subluxation. 
A further consequence of erosion of a lateral atlantoaxial joint is the loss of vertical support of the atlas on that side. The atlas therefore settles into the eroded joint and thereby undergoes a lateral tilt (Fig. 8D). This is the basis for the lateral tilt of the atlas seen in the present specimen and that detected radiographically in the cases of Burry et al. ${ }^{1}$ It is also probably the underlying cause of the compensatory scoliosis described by Bunton et al. ${ }^{2}$

A significant aspect of this analysis is that, to produce even maximal lateral subluxation of the atlas, the extent of erosion of the lateral atlantoaxial joint does not have to be great. Full cartilage erosion with as little as $1 \mathrm{~mm}$ of bony erosion of the articular processes of the joint permits the lateral mass of the atlas to subluxate as far as the odontoid process (Fig. $8 \mathrm{C})$.

It might be expected that erosion of the lateral mass should weaken the attachment of the transverse ligament of the atlas. However, the site of attachment of this ligament lies some $4 \mathrm{~mm}$ above the lateral atlantoaxial joint space. It is therefore sufficiently displaced to be not necessarily involved in slight erosive lesion of the lateral mass that otherwise might be sufficient to permit lateral subluxation (Fig. 8). Nevertheless it is acknowledged that if there is severe erosion of the lateral mass the transverse ligament could eventually be weakened, in which case anterior subluxation would become a potential complication. In this context it is worth noting that, although absent in the present specimen, concomitant anterior subluxation of the atlas was evident in 3 of the cases reported by Burry et al. ${ }^{1}$

It is important to specify that the present discussion relates strictly to pure lateral subluxation. Clinically this condition should be distinguished from rotary subluxation, which may resemble lateral subluxation. It is for this reason that it was emphasised that the present case represented an example of true lateral subluxation.

During rotation of the atlas the ipsilateral lateral mass moves forwards, and its inferior articular process may overlap the superior articular process of the axis. Concurrently the opposite lateral mass moves backwards and medially. In anteroposterior radiographs this movement resembles lateral subluxation. This illusion, however, is produced by the projection used. The contralateral mass does not in reality approximate to the odontoid process along a coronal plane. Rather, in its medial, but posterior, position $x$-rays directed anteroposteriorly project its position closer to the odontoid process. Further discussions of this phenomenon and its distinction from true lateral subluxation are available. ${ }^{4-11}$

A final issue which bears consideration is the clinical significance of lateral subluxation. Essentially the condition indicates only severe erosive arthritis. With respect to neurological complications the risk of spinal cord compression is not as great as with ventral subluxation. From the foregoing anatomical discussions it is evident that in true lateral subluxation the odontoid process eventually will limit displacement of the atlas. Provided the odontoid process and atlas remain intact, the extent of lateral subluxation cannot be great enough to compromise the spinal cord. Thus in a patient with lateral subluxation of the atlas neurological complications, at least on theoretical grounds, would not be expected. This prognosis, however, depends critically on the sustained integrity of the odontoid process and of the atlas itself and on the absence of concurrent ventral subluxation. Attention therefore should be directed to these 3 factors when monitoring the progress of patients with lateral subluxation.

\section{References}

1 Burry H C, Tweed J M, Robinson R G, Howes R. Lateral subluxation of the atlanto-axial joint in rheumatoid arthritis. Ann Rheum Dis 1978; 37: 525-8.

2 Bunton R W, Grennan D M, Palmer D G, de Silva R T D. Lateral subluxation of the atlas in rheumatoid arthritis. $\mathrm{Br} J$ Radiol 1978; 51: 963-7.

3 Corrigan A B. Radiological changes in rheumatoid cervical spines. Australas Radiol 1969; 13: 370-4.

4 Coutts M B. Atlanto-epistropheal subluxations. Arch Surg 1934; 19: 297-311

5 Evans W A. Pathologic dislocations at the atlanto-axial joint: an unusual complication of rheumatic fever. Radiology 1941; 37: 347-50.

6 Foxe A N, Friedman L J. Simple lateral luxation of the atlas. Am J Surg 1930; 8: 831-3.

7 Hohl M, Baker H R. The atlanto-axial joint. Roentgenographic and anatomical study of normal and abnormal joints. $J$ Bone Joint Surg 1964; 46A: 1739-52.

8 Jacobson G, Adler D C. An evaluation of lateral atlanto-axial displacement in injuries to the cervical spine. Radiology 1953; 61: 355-62.

9 Jacobsen G, Adler D C. Examination of the atlanto-axial joint following injury, with particular emphasis on rotational subluxation. AJR 1956; 76: 1081-94.

10 Paul L W, Moir W W. Non-pathological variations in relationship of the upper cervical vertebrae. AJR 1949; 62: 519-24.

11 Wortzman G, Dewar F P. Rotary fixation of the atlanto-axial joint: rotational atlantoaxial fixation. Radiology 1968; 90: 479-87. 